\title{
A Novel Gene Affecting the Timing of Puberty
}

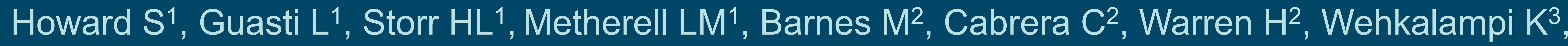
Cariboni $\mathrm{A}^{4}$ and Dunkel $\mathrm{L}^{1}$

${ }^{1}$ Centre for Endocrinology, ${ }^{2}$ William Harvey Research Institute, QMUL

${ }^{3}$ Children's Hospital, Helsinki University Central Hospital and University of Helsinki, ${ }^{4}$ Institute of Ophthalmology, UCL

\section{Background - Puberty}

-Puberty is the normal developmental stage when reproductive capacity is attained

-Disturbances of pubertal timing affect over $4 \%$ of the population -Deranged pubertal timing has significance for public health in view of the association between early or late puberty and an adverse cardiovascular, metabolic and cancer risk profile ${ }^{1-4}$

\section{Background - Puberty Genetics}

- The timing of pubertal onset has high heritability; $60-80 \%$ of variation is determined by genetic factors ${ }^{5}$ - however, the majority of these factors remain elusive

-We hypothesise that low-frequency, high or intermediate-impact variants will be enriched in populations at the extremes of normal pubertal timing (Figure 1).

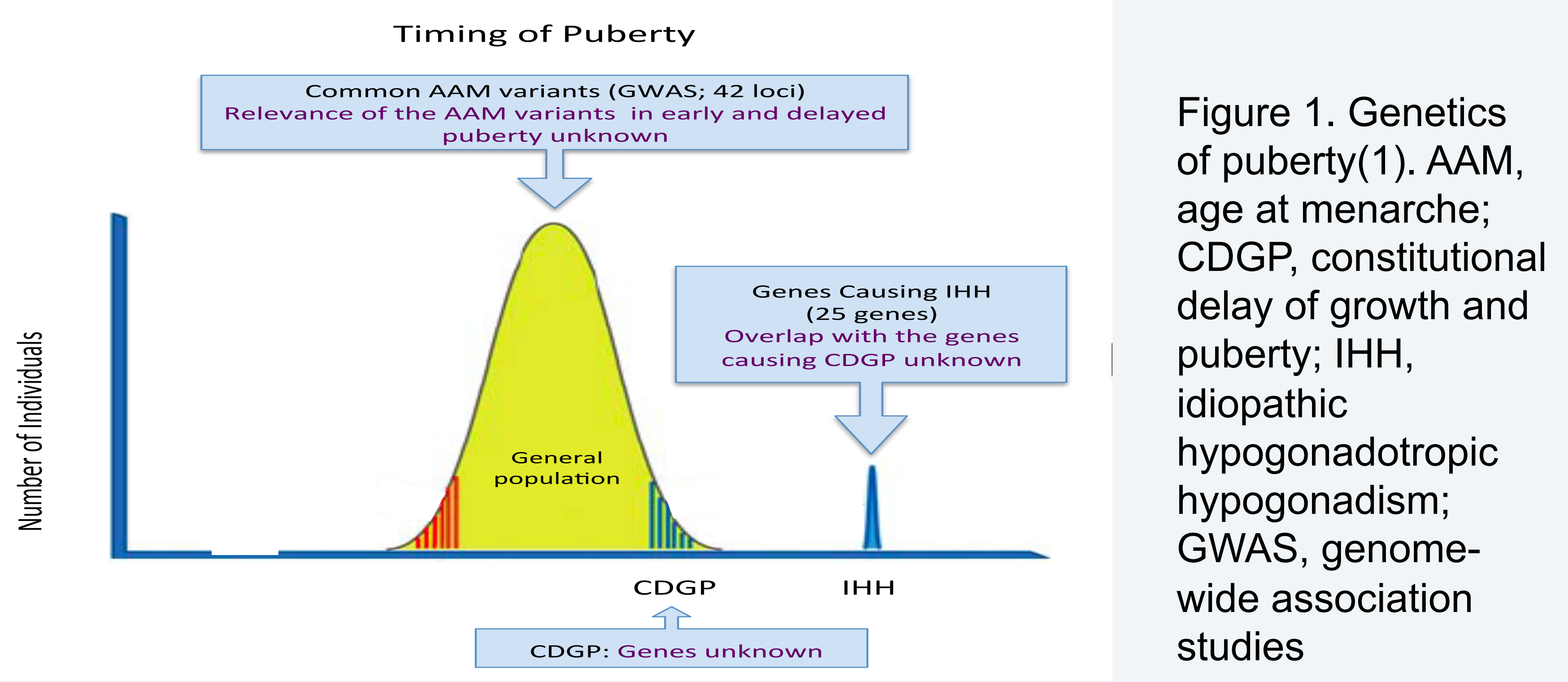

CDGP

-Familial Constitutional Delay in Growth and Puberty (CDGP) is a condition of healthy individuals with pubertal onset delayed by more than 2 standard deviations and has repeatedly been shown to cluster in families ${ }^{6}$

- Our cohort was collected from patients seen under specialist

Paediatric care from Finland between 1982-2004

- Cohort contains 403 affecteds from 170 families and their unaffected relatives (total of 910 individuals)

\section{Methods}
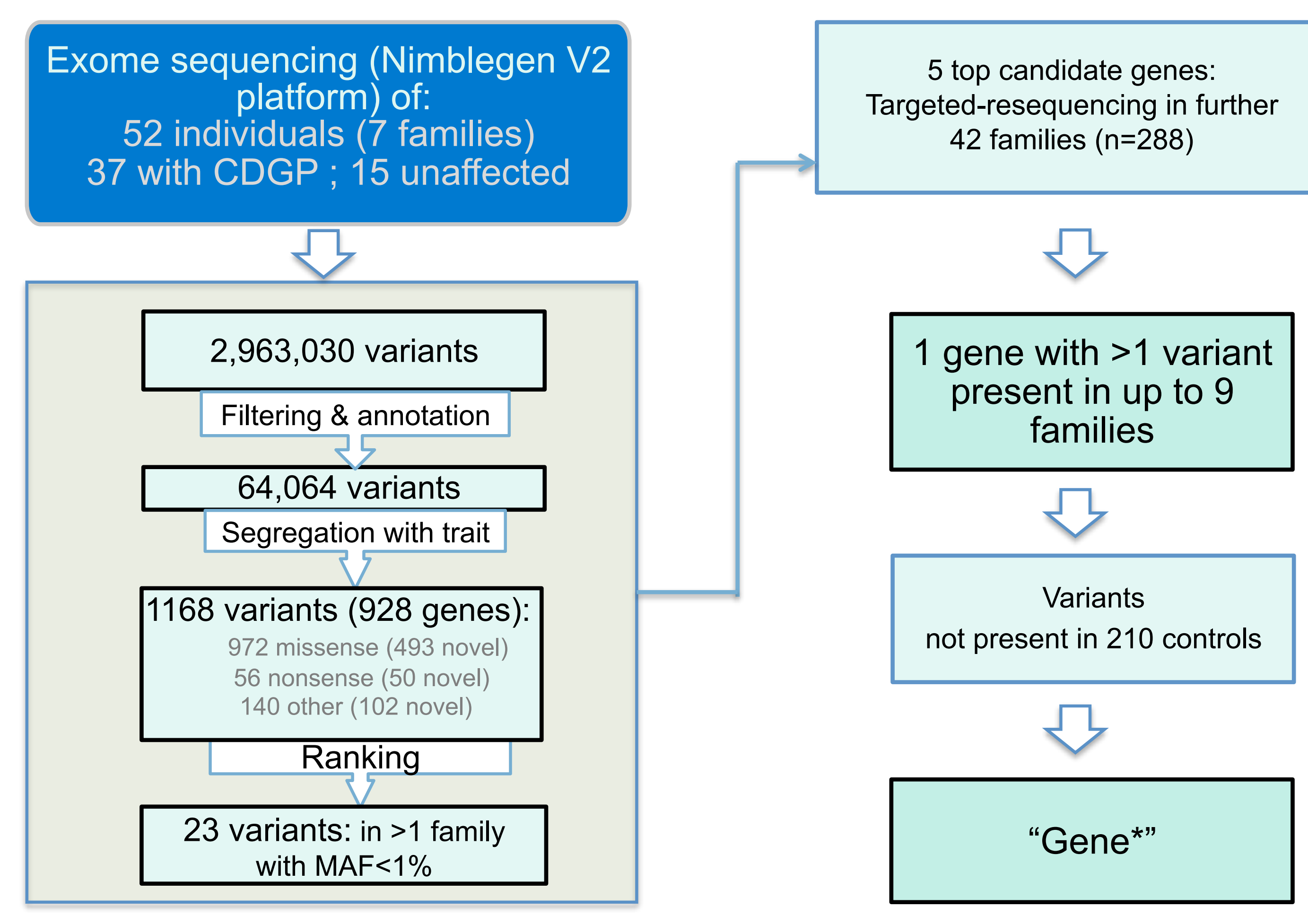

Figure 2. 'Unbiased' methodological approach to identify novel candidate genes associated with trait in our cohort of CDGP patients through next generation sequencing and filtering, via in house bioinformatic pipeline (M. Barnes unpublished) and pathway analysis.

\section{References}

Mouritsen, A., Aksglaede, L., Sorensen, K., Mogensen, S.S., Leffers, H., Main, K.M., Frederiksen, H., Andersson, A.M., Skakkebaek, N.E., and Juul, A. 2010. Int J Androl 33:346-359

Purdie, D.M., and Green, A.C. 2001. Clinical obstetrics \& gynaecology 15:341-354

He, C., Zhang, C., Hunter, D.J., Hankinson, S.E., Buck Louis, G.M., Hediger, M.L., and Hu, F.B. 2010. American journal of epidemiology 171:334-344.

Lakshman, R., Forouhi, N.G., Sharp, S.J., Luben, R., Bingham, S.A., Khaw, K.T., Wareham, N.J., and Ong, K.K. 2009 The Journal of clinical endocrinology and metabolism 94:4953-4960.

Gajdos, Z.K., Hirschhorn, J.N., and Palmert, M.R. 2009 Curr Opin Endocrinol Diabetes Obes 16:16-24

Wehkalampi, K., Widen, E., Laine, T., Palotie, A., and Dunkel, L. 2008. J Clin Endocrinol Metab 93:723-728.

\section{Results}

This strategy has identified an important candidate gene $\left(G^{*} e^{*}\right)$ : -Four variants in this gene segregate with trait in 9 families of our cohort (Figure 3).

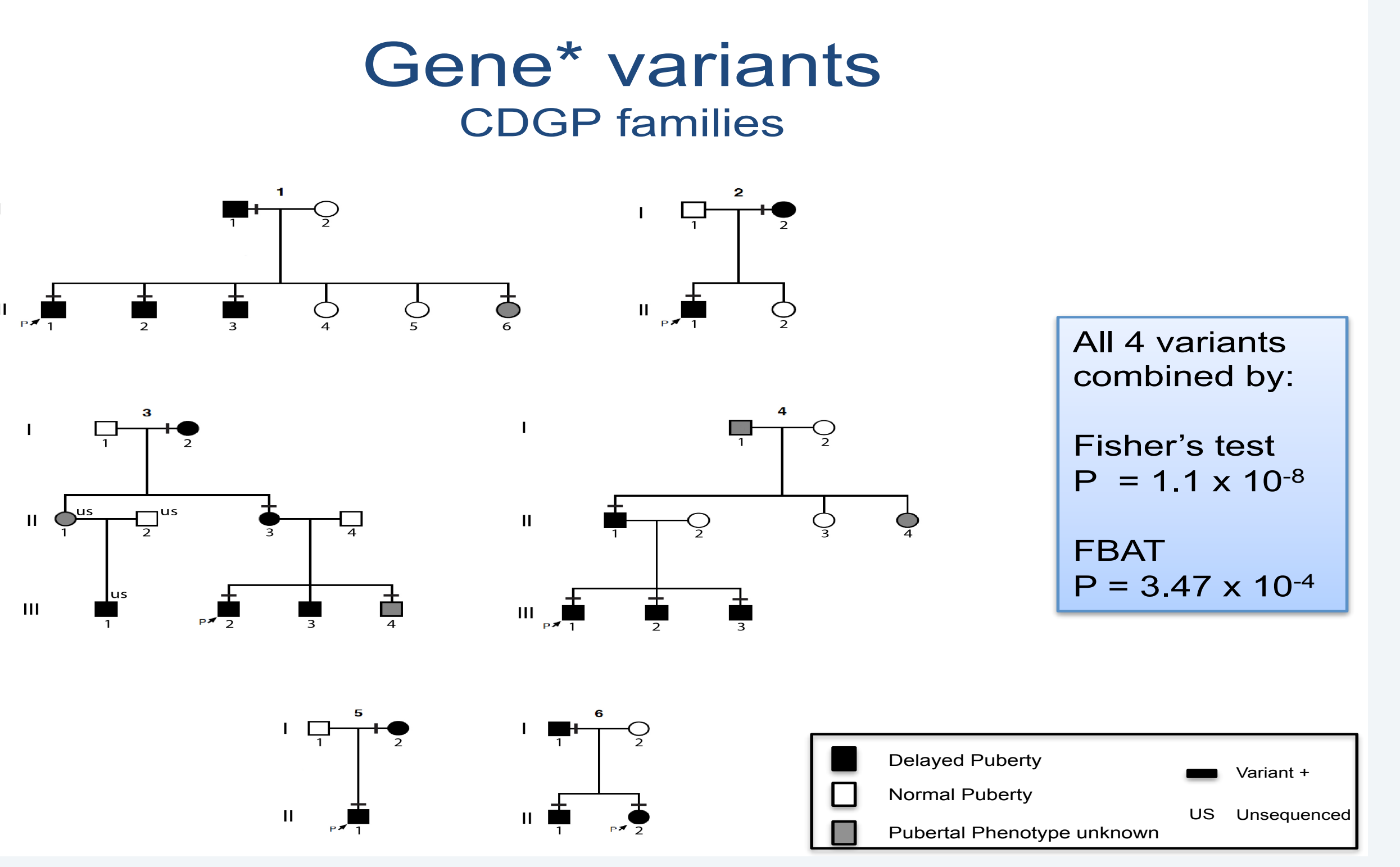

Figure 3. Pedigrees of six of nine families with CDGP with Gene* variants segregating with trait. Phenotypic symbols listed in the key, presence of Gene* variant indicated by solid black bar adjacent to individual. Statistical validation shown in blue box.

-Mutations in Gene* have not previously been described in humans -All CDGP variants fall within important domains with predicted functions of protein-protein interaction or cell adhesion (Figure 4). -Additional sequencing has identified stop-gain variants in two patients with $\mathrm{GnRH}$ deficiency from a separate cohort.

Gene* variants



-Mouse embryo studies show expression of this gene in the nasal mesenchyme, in the region where $\mathrm{GnRH}$ neurons begin their migration to the hypothalamus (Figure 5)


Figure 5A\&B. In situ hybridisation demonstrating staining for candidate gene probe (purple) within the nasal mesenchyme adjacent to the vomeronasal organ; $5 \mathrm{~A}$ mouse E12.5, 5B mouse E14.5. VNO: vomeronasal organ; NS: nasal septum; NM: nasal mesenchyme; GnRH neurons labelled with black arrows

\section{Conclusions}

-We describe a novel, in-house bioinformatic pipeline for identification of novel causal variants from next-generation sequencing data in common, complex traits

-We have identified an exciting new gene implicated in the timing of puberty, which appears to play a role in migration of $\mathrm{GnRH}$ neurons towards the hypothalamus during embryonic development.

-We demonstrate potential overlap between simple delayed puberty and hypogonadotropic hypogonadism/ Kallmann syndrome 